\title{
Progressive leptomeningeal fibrosis: a clinico-pathological case report
}

\author{
DAVID CHALIF, LEO W DUCHEN, JOHN MARSHALL, RICHARD HAYWARD
}

From the Departments of Clinical Neurology, Neuropathology, and Neurosurgery, Institute of Neurology, The National Hospital for Nervous Diseases, Queen Square, London, UK

SUMmARY A female patient developed persistent facial pain beginning at age 19 years. Intermittent motor and sensory disturbances referable to one hemisphere began nine years later and by the age of 41 she had developed signs of increased intracranial pressure. Exploratory craniotomy revealed replacement of the leptomeninges by thick, fibrous tissue. The histological appearance was that of a chronic, benign and minimally infiltrative process with a mild, non-specific inflammatory component, underlying cortical ischaemic changes, and white matter oedema. The lesion resembled nodular fasciitis, a soft tissue process. No cause of the reactive fibrosis of the meninges in this case is known.

Benign fibrous lesions occur commonly in the soft tissues yet rarely in the CNS or meninges. The reported cases of intracranial fibroblastic proliferative processes form a spectrum histopathologically, and usually present as well defined discrete tumours. ${ }^{1}$ Leptomeningeal fibrosis may follow cranial irradiation ${ }^{2}$ and sub-arachnoid haemorrhage ${ }^{3}$ but does not appear to have been reported as an idiopathic entity. We report a case of diffuse fibrosis of the leptomeninges of the right hemisphere in an otherwise healthy 41-year-old female who was aware of progressive symptoms referable to that hemisphere for more than 20 years.

\section{Case report}

The patient is a 41-year-old right handed female surveyor, who first came to attention at the National Hospital, Queen Square in 1972. She had suffered from persistent right facial pain in the first two divisions of the fifth cranial nerve for over 20 years, at times associated with rightsided headache. For four years she had experienced episodes of numbness lasting several days, over the left side of the body associated with vague clumsiness of the left hand. There was one isolated episode of loss of consciousness associated with a numb sensation in the left leg. Neurologic examination and general physical examination were negative. EEG and EMG were normal. A radio-

Dr D Chalif s present address, and address for reprint requests: Department of Neurosurgery, New York University-Bellevue Medical Center, 550 First Avenue, New York, NY 10016, USA.

Received 28 September

Accepted 29 October 1982 isotope brain scan showed an area of increased uptake in the right parietal region, however four-vessel angiography was normal. The patient was discharged with a presumptive diagnosis of migraine.

She presented next in 1976 with clumsiness of the left arm starting acutely several weeks prior to admission and slowly subsiding. In the interim period (1972-76), the left-sided motor-sensory phenomena had subsided, but the right facial pain had persisted. Neurologic examination showed left-sided hyperreflexia and mild weakness. There was no papilloedema. Lumbar puncture yielded clear colourless fluid with an opening pressure of $140 \mathrm{~mm}$ water, no cells and a normal biochemical profile. There was no oligoclonal pattern. CT scan (fig 1a) showed a low density area in the right parietal lobe which enhanced with contrast. No mass effect was appreciated. EEG demonstrated a right anterior quadrantic disturbance; regional cerebral blood flows were normal. Right carotid angiography showed small abnormal tortuous vessels over the right posterior frontal convexity, supplied mainly by a dilated anterior middle meningeal artery. The angiographic picture was thought to be consistent with low grade glioma. As the patient had minimal neurologic deficit and as the lesion was ill-defined, surgical exploration was deferred, and she was discharged to be followed as an out-patient.

The patient was re-admitted in 1981 for investigation. The right-sided facial pain had remained constant although the right-sided headache had increased in severity and would at times wake her at night. About two weeks prior to admission she had developed a slowly progressive leftsided numbness and she continued to have intermittent episodes of left-sided motor and sensory disturbances. There were no other symptoms. Family and past medical history were non-contributory. Over the previous 20 years the patient had taken a number of drugs for relief of headaches and facial pain, including aspirin, codeine phos- 

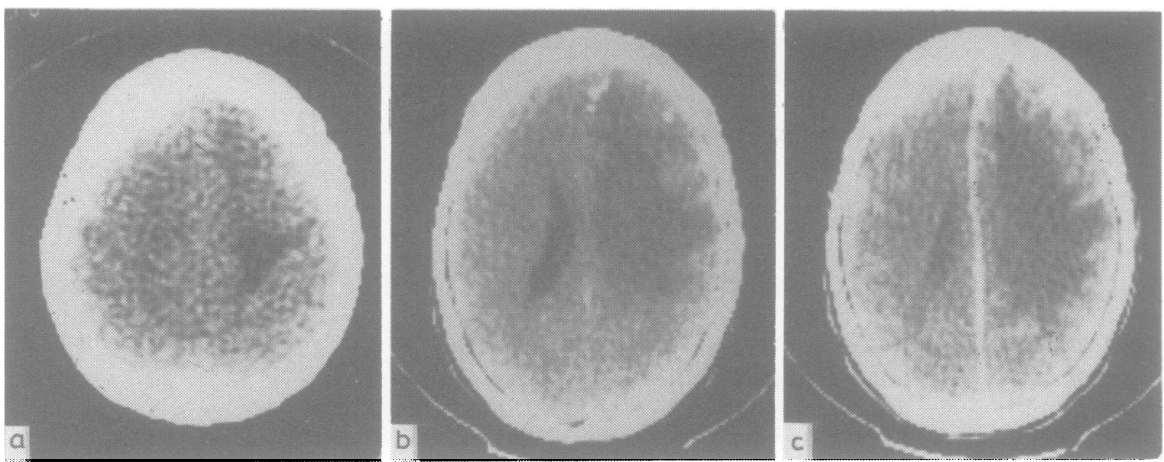

Fig 1 The non-contrast computed tomographic scan in $1976(A)$ demonstrates a small low density area in the right fronto-parietal region. By $1981(B)$ this low density area has spread to the entirety of the right frontal and parietal lobes. The low density is confined to the white matter and extends down to the internal capsule. There are small areas of calcification visible anteriorly and there are further high density areas in or adjacent to the parietal cortex. After intravenous contrast infusion (C) selective cortical enhancement is noted, along with a slight right to left shift.

phate, paracetamol, propoxyphene and benzodiazepines. Specifically, there was no history of methysergide ingestion.

Examination revealed an alert, intelligent woman with mild pyramidal weakness and hyper-reflexia on the left side. Plantar responses were flexor. There was mild blurring of the optic discs and fluorescein retinal angiography showed early papilloedema. Repeat CT scan (figs 1b, c) demonstrated extensive areas of low density in the right hemisphere, with marked cortical enhancement after contrast infusion. Bilateral carotid angiography disclosed diffuse swelling of the right cerebral hemisphere. The areas of abnormal circulation in the right posterior frontal region were persistent and the contribution of the meningeal circulation was more apparent than previously.

The patient was started on steroids with some clinical improvement and underwent right frontal craniotomy for biopsy and decompression. The surface of the brain was grossly abnormal and were covered in most areas with a 3-4 mm thick layer of firm, pinkish tissue which was lightly adherent to the overlying dura. Dilated meningeal arteries were seen in the dura adjacent to the thickened leptomeninges. In some areas where the brain was not covered by the abnormal tissue the arachnoid had a white discoloration and was thickened along some $e_{\mid}$of the sulci.

Following operation the patient made an excellent recovery. Treatment with steroids continued for several weeks during which time there was a continuing improvement in her left sided weakness. She was then able to exist without steroid therapy for several months but approximately one year after her operation she was re-admitted with a short history of deterioration during which her hemiplegia had worsened. At the time of her arrival at hospital some spontaneous improvement had started. Treatment with steroids was started once again and continued for several weeks. Further CT scans performed during this recurrence of her symptoms at first showed increased oedema in the right hemisphere but later scans demonstrated resolution of this oedema. At the time of writing she is back at work with only a slight increase in her hemiplegia compared with that noted prior to her operation.

\section{Pathology}

The operative specimen received consisted of brain tissue $1.5-2.0 \mathrm{~cm}$ in thickness including the frontal pole and adjacent gyri with underlying white matter. Many blocks were prepared so that the whole specimen has been embedded in paraffin wax. A wide variety of staining methods were used. The description which follows applies to every block studied with only minor variations. There was generalised thickening of the leptomeninges varying from about 100 $\mu \mathrm{m}$ in thickness over some gyral convexities (fig 2) up to $1.5-2.0 \mathrm{~mm}$ in the sulci. In places the leptomeninges were cellular, composed of plump fibroblast-type cells (fig 3 ) lying in a loose reticulin-rich matrix and showing moderate numbers of mitotic figures. There were scattered clusters of lymphocytes, some around vessels, but no necrosis, granulomas or plasma cells. No organisms were identified with appropriate staining techniques for bacteria or fungi. In other places the meninges were densely collagenous with few cells, and here and there small vessels and collagen had an eosinophilic hyaline appearance (not positive for amyloid). Arterial and venous vessels were present within the thickened leptomeninges as well as endothelium-lined channels. Although vessels were numerous in some places, particularly in sulci, the tissue was not vascular enough to be considered angiomatous and only occasional granules of haemosiderin pigment were present, too little to indicate previous bleeding. Vessels were surrounded by concentric layers of collagen and some seemed to be constricted by the dense surrounding tissue. Several vessels were seen which have a greatly narrowed lumen because of intimal thickening and smooth muscle proliferation suggesting previous occlusion and recanalisa- 


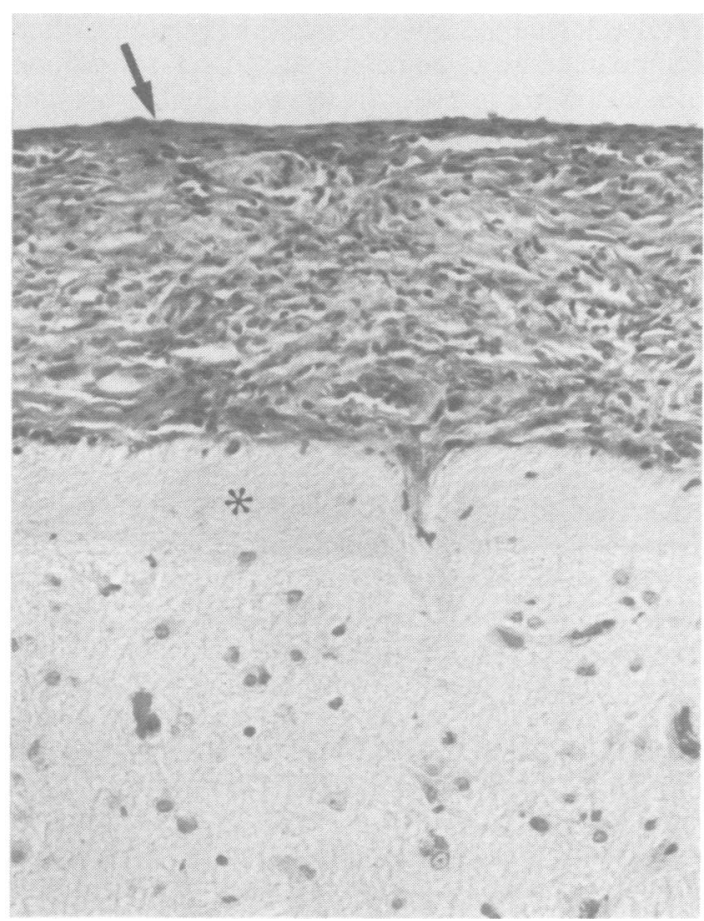

Fig 2 Section showing meninges and underlying cerebral cortex at the convexity of a gyrus. There is marked thickening of the pia-arachnoid (arrow) which is here about $200 \mu \mathrm{m}$ thick, densely collagenous with a scattering of lymphocytes. There are no granulomas. Immediately below the pia there is a broad (about $80 \mu \mathrm{m}$ ) band of glial fibres $\left({ }^{*}\right)$ and the molecular layer beneath shows reactive astrocytic gliosis. (Haematoxylin and eosin $\times 40$.)

tion. There was no arteritis or phlebitis and no acute thrombosis. Perivascular fibrosis was seen around some vessels in the white matter (fig 4) and may represent an extension of the fibrous lesion along Virchow-Robin spaces.

The underlying brain showed changes which could almost certainly all be interpreted as secondary to the meningeal and vascular pathology. A layer of dense astrocytic glial fibres, strongly stained by the Holzer method, lay immediately beneath the pia and occupies the superficial $100-200 \mu \mathrm{m}$ of the molecular layer. The remainder of the molecular layer showed an increased number of astrocytes and in places these were of the reactive type with abundant eosinophilic cytoplasm. Several areas of cortex showed ischaemic changes, the most severe being around the depths of some sulci with laminar necrosis, capillary and glial proliferation and abundant fat granule cells. One of these areas of cortical necrosis was much older than the others and shows abundant collagen formation within the softened zone. White matter beneath the necrotic areas of cortex showed astrocytic gliosis, some myelin loss and, in places, oedema.

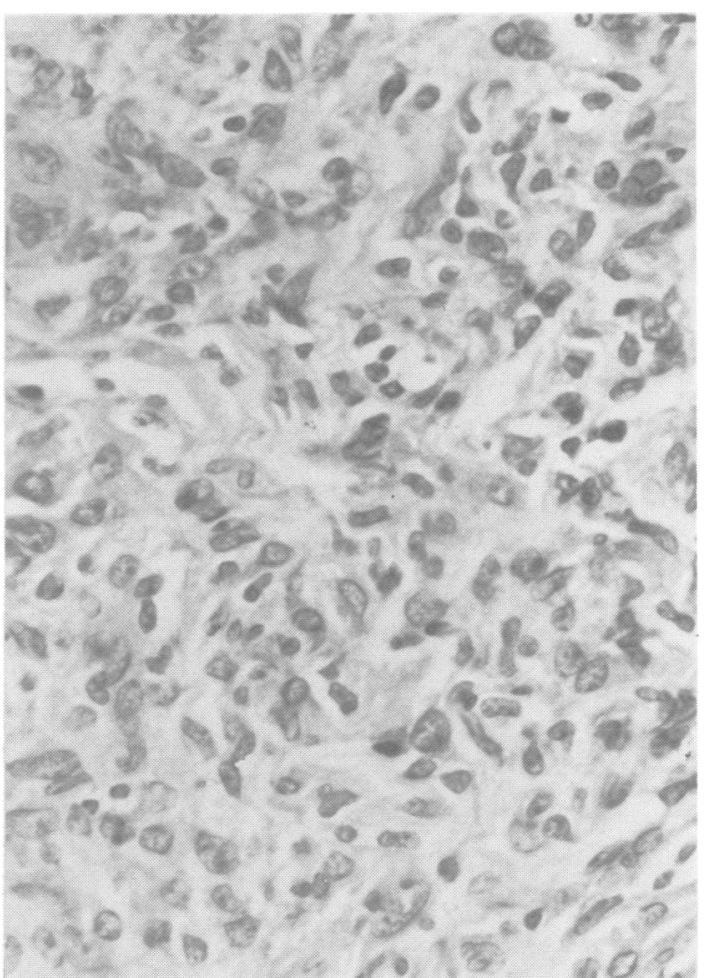

Fig 3 Section showing a cellular area of meninges.

Fibroblasts and occasional lymphocytes lie in a loose matrix which, with appropriate staining, can be shown to be rich in reticulin fibres but contains little collagen. (Haematoxylin and eosin $\times 100$.)

\section{Discussion}

Benign lesions composed of fibrous elements, commonly termed fibrous dysplasias, desmoids, fibromas, and fibromatoses are exceedingly rare in the CNS and meninges. A review of the differential diagnosis of fibrous intracranial lesions ${ }^{1}$ indicates that the lesion we report is histologically unique.

Benign fibrous proliferations make up a variety of soft tissue masses and are generally classified into a group of lesions known as the fibromatoses. The fibromatoses form a spectrum of processes and are best defined as locally invasive, idiopathic, nonmetastasising fibrous tumours which are of mesenchymal origin and recurrent after surgical excision..$^{4-6}$ Among the fibromatoses are entities such as palmar fibromatosis, Dupuytren type fibromatosis, and congenital generalised fibromatosis. Histologically these lesions are viewed as neoplastic tissue which continues to proliferate, thereby becoming an 


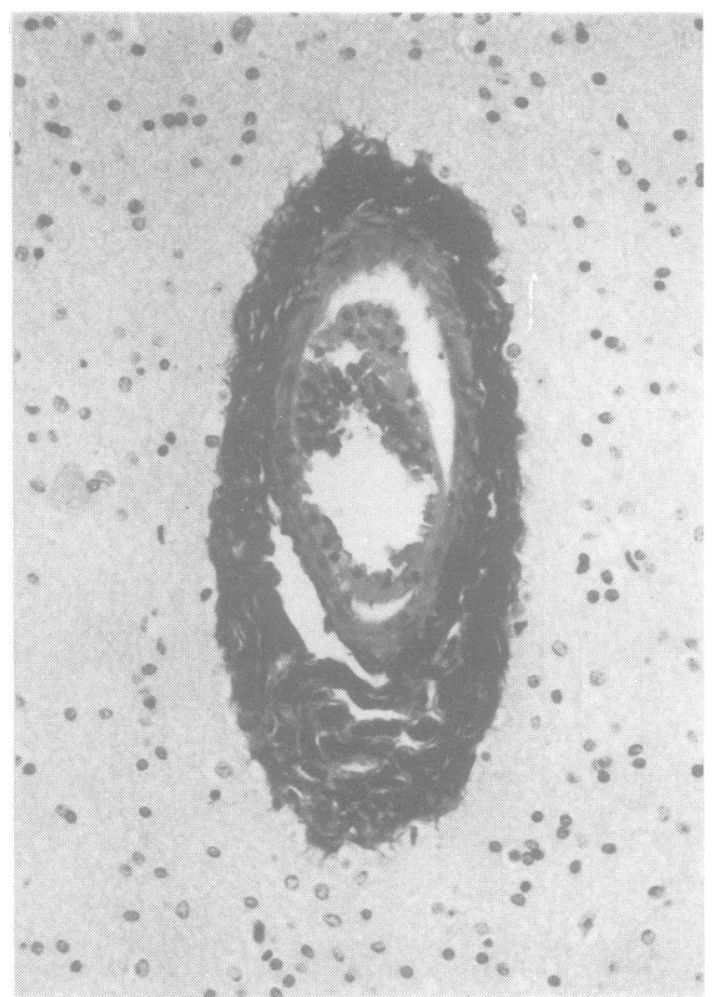

Fig 4 Section through gliotic white matter. A small blood vessel, probably venous, is shown surrounded by a thick peri-vascular layer of dense collagen. (Haematoxylin and van Gieson $\times$ 100.)

infiltrating local mass. Proliferation of fibroblastic elements can also be classified as reactive or inflammatory phenomena, ${ }^{5}$ as in the case of postirradiation fibrosis, ${ }^{27}$ post subarachnoid haemorrhage fibrosis ${ }^{3}$ and nodular fasciitis. ${ }^{8}$ The histopathologic and aetiologic variability of benign fibrous lesions make strict classification difficult. It appears however, that the leptomeningeal fibrosis we report has properties of both the fibromatoses and the reactive fibrous lesions.

To date there are less than 20 reports in the literature of benign CNS and meningeal fibromas or fibromatoses, and these form a variety of lesions ranging from the so-called fibroblastic meningioma to post-irradiation intracranial fibromas. ${ }^{29-19}$ Histologically these tumours were somewhat variable; however, each showed dense growth of fibrous tissue and stained densely for collagen and reticulin. Few mitoses were noted and there were no reports of metastases. It is of note that except for one isolated report of dural fibrosis presenting as infantile spasms, ${ }^{14}$ all other CNS fibrous lesions were dis- crete, well circumscribed intracranial tumours, often accompanied by a significant mass effect. In addition to hemispheric masses, an intra-cerebellar lesion ${ }^{17}$ and an intramedullary thoracic lesion ${ }^{1}$ have been observed. Fibrous tumours of bone, ossifying fibromas, are also known to occur intracranially, arising from the craniofacial bones. ${ }^{20-22}$ In contrast, the fibrous process in the present case is not tumorous in character and is confined to the leptomeninges and adjacent vessels. Macroscopically, the leptomeninges were thickened at surgery and histopathologically the lesion shows a diffuse proliferation of fibroblastic elements in the leptomeningeal plane.

Radiographically, pan-hemispheric low density was noted and the unusual feature of selective cortical enhancement extending from frontal to occipital lobes suggests that the process spread diffusely through the leptomeninges over the right hemisphere. The low density, which had been progressive since the previous scan, may reflect oedema and reaction to widespread zones of laminar cortical infarction which were found histologically. These zones of infarction are thought to be a result of perivascular fibrosis and the occlusion of vessels in the sub-arachnoid space and cortex. Arteritis was not noted histologically, but dilatation of the meningeal vessels was seen both on angiography and at surgery.

The lesion appeared to be chronic histologically and survey of the specimen showed varying degrees of density and organisation of the fibrous cellular elements. The symptom of right sided facial pain may indicate that this lesion was clinically long standing, as the pain could represent chronic trigeminal irritation or the effect of the documented engorgement of right hemispheric meningeal vessels.

The fibrotic leptomeninges are strikingly similar histologically to the soft tissue mass termed nodular fasciitis. This is a fascial mass composed of lobules of fibroblasts, capillaries, a scant chronic inflammatory cell infiltrate and areas representing granulation tissue in a myxoid stroma or collagen matrix $.^{83} \mathrm{Nodu}-$ lar fasciitis has been called a "reactive" phenomenon in the soft tissues. ${ }^{8}$ In the case reported, although there was sub-pial gliosis, there was no clear source of meningeal irritation. Additionally, although this leptomeningeal lesion is histologically similar to nodular fasciitis, the clinical characteristics differ and here the lesion appeared to be somewhat more locally infiltrative. Although there is no evidence of direct extension into the cortex, perivascular fibrosis was noted in both grey and white matter subjacent to the thickened leptomeninges. This fibrosis seems to occupy the perivascular Virchow- 
Robin spaces. The presentation differs also, as the patient reported here had a slowly progressive 24 year history, while the reactive fibroses commonly have a much shorter clinical course.

Radiation therapy and chemotherapeutic agents ${ }^{24}$ have been implicated as aetiological factors causing aberrent growth of fibrous tissue. Quest $e t$ $a l^{2}$ report excision of a frontal fibrous tumour following cranial radiation therapy and CNS fibrosarcoma is well documented following irradiation. ${ }^{25}$ Methysergide, an ergotamine derivative used as an antimigraine agent, has been implicated as a cause of retro-peritoneal fibrosis. ${ }^{24}$ There is, however, no evidence in the literature of drug induced intracranial fibrosis in man. Subarachnoid haemorrhage is well known to cause fibrosis of the leptomeninges, ${ }^{3}$ usually basal, often leading to communicating hydrocephalus. In this case there was no history or clinical evidence of subarachnoid haemorrhage, irradiation, trauma, infection or the use of methysergide.

It is difficult to categorise formally this idiopathic leptomeningeal fibrosis. Developmentally, it is presumed that fibrous tumours of the CNS arise from mesenchymal precursors in the leptomeninges and dura or from perivascular connective tissue, and have therefore been called "congenital neoplasms" by Koos et al, ${ }^{16}$ rather than true neoplasms. A fibroma involving the dura has been described in a five month old child with congenital generalised fibromatosis. ${ }^{26}$ Conditions such as tuberous sclerosis and neurofibromatosis, which are associated with a proliferation of fibrous elements within the CNS, must be considered when interpreting the pathology in this case although the patient had none of the stigmata of these disorders.

We thank Professor du Boulay for the neuroradiological reports and for figure 1.

\section{References}

${ }^{1}$ Friede RL, Pollak A. Neurosurgical desmoid tumorspresentation of four cases with a review of the differential diagnosis. $J$ Neurosurg 1979;50:725-32.

${ }^{2}$ Quest DO, Salcman M. Fibromatosis presenting as a cranial mass lesion. Case report. J Neurosurg 1976; 44:237-40.

${ }^{3}$ Suzuki P, Ishii M, Ottomo M, et al. Changes in the subarachnoid space after experimental subarachnoid haemorrhage in the dog. Scanning electron microscopic observations. Acta Neurochir 1977;39:1-14.

${ }^{4}$ Allen PW. The fibromatoses-a clinicopathologic classification based on 140 cases. Am J Surg Path 1977;1:255-70.

${ }^{5}$ Mackenzie DH. The differential diagnosis of fibroblastic disorders. Oxford: Blackwell Scientific Publications, 1970.
${ }^{6}$ Stout AP. Tumours of the soft tissues. In: Atlas of Tumour Pathology, Section 2, fascicle 5: Armed Forces Institute of Pathology, Washington DC, 1967.

${ }^{7}$ Petit UD, Chamness JT, Ackerman LV. Fibromatosis and fibrosarcoma following irradiation therapy. Cancer 1954;7:149-58.

${ }^{8}$ Price EB, Silliphant WM, Schuman R. Nodular fasciitis: A clinico-pathologic analysis of 15 cases. Am J Clin Pathol 1961;35:122-36.

9 Alpers BJ, Grant FC, Yaskin J. Primary fibroblastoma of the brain. Arch Neurol Psychiat 1931;26:1335 (abstract).

${ }^{10}$ Amezua L. Fibroma intracerebral gigante recidivado Hibernacion artificial. Acta Neuropsiquiat Argent 1958;4:15-22.

1 Baker AB, Adams JM. Primary fibroblastoma of the brain. Report of a case. Am J Pathol 1937;13:129-37.

${ }^{12}$ Brucher JM, Hizawa K, Wechsler W. Ultrastructure d'un fibrome intracerebral et possibilité de transformation maligne de ce type tumoral. Rev Neurol (Paris) 1970;123:443-5.

${ }^{13}$ Carrillo R, Ricoy JR, Herrero-Vallejo J, et al. Fibrous xanthomas of the brain. Report of two cases. Clin Neurol Neurosurg 1975;78:34-40.

${ }^{14}$ Dolman CL, Crichton JU, Jones EA, et al. Fibromatosis of the dura presenting as infantile spasms. J Neurol Sci 1981;49:31-9.

${ }^{15}$ Kepes JJ, Kepes M, Slowick F. Fibrous xanthomas and xanthosarcomas of the meninges and brain. Acta Neuropathol 1973;23:187-99.

${ }^{16}$ Koos WJ, Jellmeyer K, Sunder-Plassmann M. Intracerebral fibroma in an 11 month old infant. Case report. J Neurosurg 1971;35:77-81.

${ }^{17}$ Llena JF, Chung HD, Hirano A, et al. Intracerebellar "fibroma". Case report. J Neurosurg 1975;43:98101.

${ }^{18}$ Scott M, Peale AR, Croissant PD. Intracranial midline anterior fossae ossifying fibroma invading orbits, paranasal sinuses, and right maxillary antrum. Case report. J Neurosurg 1971;34:827-31.

${ }^{19}$ Zulch KJ. Fibrome. In: Olivecrona H, Tonnis W, eds. Handbuch der Neurochirurgie, Vol. 3. Berlin: Springer-Verlag, 1956:467.

${ }^{20}$ Kamisasa A, Kuwbara T, Inaba Y. Intracranial ossifying fibroma. Report of a case. Bull Tokyo Med Dent Univ 1973;20:209-20.

${ }^{21}$ Leheer HZ. Ossifying fibroma of the orbital roof. Its distinction from blistering or intraosseous meningioma. Arch Neurol 1969;20:536-41.

${ }^{22}$ Malcomson KG. Ossifying fibroma of the sphenoid. $J$ Laryng 1967;81:87-92.

${ }^{23}$ Hutter RVP, Stewart FW, Foote FW, Jr. Fasciitis. A report of 70 cases with follow-up proving the benignity of the lesion. Cancer 1962;15:992-1003.

${ }^{24}$ Hindle W, Pogner E, Sweetnam MT. Pleural effusion and fibrosis during treatment with methysergide. $\mathrm{Br}$ Med J 1970;1:605-6.

${ }^{25}$ Schrantz JL, Araoz CA. Radiation induced meningeal fibrosarcoma. Arch Pathol 1972;93:26-31.

${ }^{26}$ Teng P, Warden MJ, Cohn WL. Congenital generalized fibromatosis (renal and skeletal) with complete spontaneous regression. $J$ Pediatr 1963;62:748-53. 